\title{
Sums of finite products of Legendre and Laguerre polynomials
}

\author{
Taekyun Kim ${ }^{1}$, Dae San Kim², Dmitry V. Dolgy ${ }^{3}$ and Jin-Woo Park ${ }^{4^{*}}$
}

\section{"Correspondence:}

a0417001@knu.ac.kr

${ }^{4}$ Department of Mathematics

Education, Daegu University,

Gyeongsan-si, Republic of Korea

Full list of author information is

available at the end of the article

\begin{abstract}
In this paper, we study sums of finite products of Legendre and Laguerre polynomials and derive Fourier series expansions of functions associated with them. From these Fourier series expansions, we are going to express those sums of finite products as linear combinations of Bernoulli polynomials. Further, by using a method other than Fourier series expansions, we will be able to express those sums in terms of Euler polynomials.
\end{abstract}

MSC: $11 \mathrm{~B} 68 ; 33 \mathrm{C} 45 ; 42 \mathrm{~A} 16$

Keywords: Fourier series; Finite products; Legendre polynomials; Laguerre polynomials; Bernoulli polynomials; Euler polynomials

\section{Introduction and preliminaries}

The Coulomb potential can be written as a series in Legendre polynomials $P_{n}(x)(n \geq 0)$. They satisfy the orthogonality relation

$$
\int_{-1}^{1} P_{n}(x) P_{m}(x) d x=\frac{2}{2 n+1} \delta_{n, m}
$$

and are solutions to the Legendre equation

$$
\left(1-x^{2}\right) P_{n}^{\prime \prime}(x)-2 x P_{n}^{\prime}(x)+n(n+1) P_{n}(x)=0 .
$$

We let the reader refer to [1] for further applications of Legendre polynomials.

The Laguerre polynomials $L_{n}(x)$ have important applications to the solution of Schrödinger's equation for the hydrogen atom. They are orthogonal over the interval $[0, \infty)$ with the weight function $e^{-x}$, namely

$$
\int_{0}^{\infty} L_{n}(x) L_{m}(x) e^{-x} d x=\delta_{n, m}
$$

and are solution to the Laguerre equation

$$
x L_{n}^{\prime \prime}(x)+(1-x) L_{n}^{\prime}(x)+n L_{n}(x)=0 .
$$

(c) The Author(s) 2018. This article is distributed under the terms of the Creative Commons Attribution 4.0 International License (http://creativecommons.org/licenses/by/4.0/), which permits unrestricted use, distribution, and reproduction in any medium, provided you give appropriate credit to the original author(s) and the source, provide a link to the Creative Commons license, and indicate if changes were made. 
The Legendre polynomials $P_{n}(x)$ and Laguerre polynomials $L_{n}(x)$ are respectively defined by the recurrence relations as in the following (see [2-4]):

$$
\begin{aligned}
& (n+2) P_{n+2}(x)=(2 n+3) x P_{n+1}(x)-(n+1) P_{n}(x) \quad(n \geq 0), \\
& P_{0}(x)=1, \quad P_{1}(x)=x, \\
& (n+2) L_{n+2}(x)=(2 n+3-x) L_{n+1}(x)-(n+1) L_{n}(x) \quad(n \geq 0), \\
& L_{0}(x)=1, \quad L_{1}(x)=-x+1 .
\end{aligned}
$$

Both $P_{n}(x)$ and $L_{n}(x)$ are polynomials of degree $n$ with rational coefficients.

From (1.1) and (1.2), it can be easily seen that the generating functions for $P_{n}(x)$ and $L_{n}(x)$ are given by (see [2-4])

$$
\begin{aligned}
& F(t, x)=\left(1-2 x t+t^{2}\right)^{-\frac{1}{2}}=\sum_{n=0}^{\infty} P_{n}(x) t^{n}, \\
& G(t, x)=(1-t)^{-1} \exp \left(-\frac{x t}{1-t}\right)=\sum_{n=0}^{\infty} L_{n}(x) t^{n} .
\end{aligned}
$$

As is well known, the Bernoulli polynomials $B_{n}(x)$ are given by

$$
\frac{t}{e^{t}-1} e^{x t}=\sum_{n=0}^{\infty} B_{n}(x) \frac{t^{n}}{n !}
$$

For any real number $x$, we let

$$
\langle x\rangle=x-[x] \in[0,1)
$$

denote the fractional part of $x$, where $[x]$ is the greatest integer $\leq x$.

We recall here that

(a) for $m \geq 2$,

$$
B_{m}(\langle x\rangle)=-m ! \sum_{\substack{n=-\infty \\ n \neq 0}}^{\infty} \frac{e^{2 \pi i n x}}{(2 \pi i n)^{m}}
$$

(b)

$$
-\sum_{\substack{n=-\infty \\ n \neq 0}}^{\infty} \frac{e^{2 \pi i n x}}{2 \pi i n}= \begin{cases}B_{1}(\langle x\rangle), & \text { for } x \in \mathbb{R}-\mathbb{Z}, \\ 0, & \text { for } x \in \mathbb{Z} .\end{cases}
$$

For any integers $m, r$ with $m, r \geq 1$, we put

$$
\alpha_{m, r}(x)=\sum_{i_{1}+i_{2}+\cdots+i_{2 r+1}=m} P_{i_{1}}(x) P_{i_{2}}(x) \cdots P_{i_{2 r+1}}(x)
$$

where the sum runs over all nonnegative integers $i_{1}, i_{2}, \ldots, i_{2 r+1}$ with $i_{1}+i_{2}+\cdots+i_{2 r+1}=m$. 
Then we will consider the function $\alpha_{m, r}(\langle x\rangle)$ and derive its Fourier series expansions. As an immediate corollary to these Fourier series expansions, we will be able to express $\alpha_{m, r}(x)$ as a linear combination of Bernoulli polynomials $B_{n}(x)$. We state this here as Theorem A.

Theorem A For any integers $m, r$ with $m, r \geq 1$, we let

$$
\Delta_{m, r}=\frac{1}{(2 r-1) ! ! 2^{m+r}} \sum_{k=0}^{\left[\frac{m-1}{2}\right]}(-1)^{k}\left(\begin{array}{c}
m+r \\
k
\end{array}\right)\left(\begin{array}{c}
2 m+2 r-2 k \\
m+r
\end{array}\right)(m+r-2 k)_{r}
$$

Then we have the identity

$$
\sum_{i_{1}+\cdots+i_{2 r+1}=m} P_{i_{1}}(x) \cdots P_{i_{2 r+1}}(x)=\frac{1}{2 r-1} \sum_{j=0}^{m} \frac{(2 r+2 j-3) ! !}{(2 r-3) ! ! j !} \Delta_{m-j+1, r+j-1} B_{j}(x) .
$$

Here $(x)_{r}=x(x-1) \cdots(x-r+1)$ for $r \geq 1,(x)_{0}=1,(2 n-1) ! !=(2 n-1)(2 n-3) \cdots 1$ for $n \geq 1$, and $(-1) ! !=1$.

Also, for any integers $m, r$ with $m \geq 1, r \geq 0$, we put

$$
\beta_{m, r}(x)=\sum_{i_{1}+\cdots+i_{r+1}=m} L_{i_{1}}\left(\frac{x}{r+1}\right) L_{i_{2}}\left(\frac{x}{r+1}\right) \cdots L_{i_{r+1}}\left(\frac{x}{r+1}\right),
$$

where the sum is over all nonnegative integers $i_{1}, i_{2}, \ldots, i_{r+1}$ with $i_{1}+\cdots+i_{r+1}=m$.

Then we will study the function $\beta_{m, r}(\langle x\rangle)$ and obtain its Fourier series expansions. Again, as a corollary to these, we can express $\beta_{m, r}(x)$ in terms of Bernoulli polynomials. Here our result is as follows.

Theorem B For any integers $m, r$ with $m \geq 1, r \geq 0$, we let

$$
\Omega_{m, r}=(-1)^{m} \sum_{k=0}^{m-1} \frac{(-1)^{k}}{(m-k) !}\left(\begin{array}{c}
m+r \\
k
\end{array}\right)
$$

Then the following identity holds:

$$
\begin{aligned}
& \sum_{i_{1}+\cdots+i_{r+1}=m} L_{i_{1}}\left(\frac{x}{r+1}\right) L_{i_{2}}\left(\frac{x}{r+1}\right) \cdots L_{i_{r+1}}\left(\frac{x}{r+1}\right) \\
& =-\sum_{j=0}^{m} \frac{(-1)^{j}}{j !} \Omega_{m-j+1, r+j-1} B_{j}(x) .
\end{aligned}
$$

Here we note that neither $P_{n}(x)$ nor $L_{n}(x)$ is an Appell polynomial, whereas all our related results, except [5], have been only about Appell polynomials (see [6-9]).

Assume that the polynomials $p_{n}(x), q_{n}(x)$, and $r_{n}(x)$ have degree $n$. The linearization problem in general consists in determining the coefficients $c_{n m}(k)$ in the expansion of the product of two polynomials $q_{n}(x)$ and $r_{m}(x)$ in terms of an arbitrary polynomial sequence 
$\left\{p_{k}(x)\right\}_{k \geq 0}$ :

$$
q_{n}(x) r_{m}(x)=\sum_{k=0}^{n+m} c_{n m}(k) p_{k}(x)
$$

Thus our results in Theorems A and B can be viewed as generalizations of the linearization problem.

Our study of sums of finite products of special polynomials in this paper can be further justified by the following. Let us put

$$
\gamma_{m}(x)=\sum_{k=1}^{m-1} \frac{1}{k(m-k)} B_{k}(x) B_{m-k}(x) \quad(m \geq 2) .
$$

Just as we see in (1.10) and (1.12), $\gamma_{m}(x)$ can be expressed in terms of Bernoulli polynomials from the Fourier series expansions of $\gamma_{m}(\langle x\rangle)$. From these expansions and after some simple modification, we can obtain the famous Faber-Pandharipande-Zagier identity (see [10]) and a variant of Miki's identity (see [11-14]).

The papers $[15,16]$ are excellent sources for operational techniques. Finally, for some of the recent results, we let the reader refer to the papers [5-9, 17-19].

\section{Fourier series expansions for functions associated with Legendre polynomials}

We start with the following lemma which will play an important role in this section.

Lemma 2.1 Let $n, r$ be integers with $n, r \geq 0$. Then we have the identity

$$
\sum_{i_{1}+i_{2}+\cdots+i_{2 r+1}=n} P_{i_{1}}(x) P_{i_{2}}(x) \cdots P_{i_{2 r+1}}(x)=\frac{1}{(2 r-1) ! !} P_{n+r}^{(r)}(x),
$$

where the sum is over all nonnegative integers $i_{1}, i_{2}, \ldots, i_{2 r+1}$ with $i_{1}+i_{2}+\cdots+i_{2 r+1}=n$.

Proof By differentiating (1.3) $r$ times, we obtain

$$
\begin{aligned}
& \frac{\partial^{r} F(t, x)}{\partial x^{r}}=(2 r-1) ! ! t^{r}\left(1-2 x t+t^{2}\right)^{-r-\frac{1}{2}}, \\
& \frac{\partial^{r} F(t, x)}{\partial x^{r}}=\sum_{n=r}^{\infty} P_{n}^{(r)}(x) t^{n}=\sum_{n=0}^{\infty} P_{n+r}^{(r)}(x) t^{n+r} .
\end{aligned}
$$

From (2.2) and (2.3), we have

$$
\frac{(2 r-1) ! !}{\left(1-2 x t+t^{2}\right)^{r+\frac{1}{2}}}=\sum_{n=0}^{\infty} P_{n+r}^{(r)}(x) t^{n} .
$$


On the other hand, from (1.3) and (2.2) we observe that

$$
\begin{aligned}
& \sum_{n=0}^{\infty}\left(\sum_{i_{1}+i_{2}+\cdots+i_{2 r+1}=n} P_{i_{1}}(x) P_{i_{2}}(x) \cdots P_{i_{2 r+1}}(x)\right) t^{n} \\
& \quad=\left(\sum_{n=0}^{\infty} P_{n}(x) t^{n}\right)^{2 r+1} \\
& =\left(1-2 x t+t^{2}\right)^{-r-\frac{1}{2}} \\
& \quad=\frac{1}{(2 r-1) ! !} \sum_{n=0}^{\infty} P_{n+r}^{(r)}(x) t^{n} .
\end{aligned}
$$

Comparing both sides of (2.5), we get the desired result.

It is known that the Legendre polynomials $P_{n}(x)$ are given by (see $\left.[2,3]\right)$

$$
P_{n}(x)={ }_{2} F_{1}\left(\begin{array}{c}
-n, n+1 \\
1
\end{array} \mid \frac{1-x}{2}\right)=\frac{1}{2^{n}} \sum_{k=0}^{\left[\frac{n}{2}\right]}(-1)^{k}\left(\begin{array}{l}
n \\
k
\end{array}\right)\left(\begin{array}{c}
2 n-2 k \\
n
\end{array}\right) x^{n-2 k}
$$

where ${ }_{2} F_{1}\left(\begin{array}{c}a, b \\ c\end{array} \mid z\right)=\sum_{n=0}^{\infty} \frac{\langle a\rangle_{n}\langle b\rangle_{n}}{\langle c\rangle_{n}} \frac{z^{n}}{n !}$ is the Gauss hypergeometric function with $\langle x\rangle_{n}$ denoting the rising factorial polynomial defined by

$$
\langle x\rangle_{n}=x(x+1) \cdots(x+n-1) \quad(n \geq 1), \quad\langle x\rangle_{0}=1 .
$$

The $r$ th derivative of (2.6) is given by

$$
P_{n}^{(r)}(x)=\frac{1}{2^{n}} \sum_{k=0}^{\left[\frac{n-r}{2}\right]}(-1)^{k}\left(\begin{array}{l}
n \\
k
\end{array}\right)\left(\begin{array}{c}
2 n-2 k \\
n
\end{array}\right)(n-2 k)_{r} x^{n-2 k-r} \quad(0 \leq r \leq n)
$$

Combining (2.1) and (2.7), we get the following lemma.

Lemma 2.2 For integers $n, r$ with $n, r \geq 0$, we have the following identity:

$$
\begin{aligned}
& \sum_{i_{1}+i_{2}+\cdots+i_{2 r+1}=n} P_{i_{1}}(x) P_{i_{2}}(x) \cdots P_{i_{2 r+1}}(x) \\
= & \frac{1}{(2 r-1) ! ! 2^{n+r}} \sum_{k=0}^{\left[\frac{n}{2}\right]}(-1)^{k}\left(\begin{array}{c}
n+r \\
k
\end{array}\right)\left(\begin{array}{c}
2 n+2 r-2 k \\
n+r
\end{array}\right)(n+r-2 k)_{r} x^{n-2 k} .
\end{aligned}
$$

For integers $m, r$ with $m, r \geq 1$ as in (1.9), we let

$$
\alpha_{m, r}(x)=\sum_{i_{1}+i_{2}+\cdots+i_{2 r+1}=m} P_{i_{1}}(x) P_{i_{2}}(x) \cdots P_{i_{2 r+1}}(x) .
$$

Then we will consider the function

$$
\alpha_{m, r}(\langle x\rangle)=\sum_{i_{1}+i_{2}+\cdots+i_{2 r+1}=m} P_{i_{1}}(\langle x\rangle) P_{i_{2}}(\langle x\rangle) \cdots P_{i_{2 r+1}}(\langle x\rangle)
$$


defined on $\mathbb{R}$, which is periodic with period 1 . The Fourier series of $\alpha_{m, r}(\langle x\rangle)$ is

$$
\sum_{n=-\infty}^{\infty} A_{n}^{(m, r)} e^{2 \pi i n x}
$$

where

$$
\begin{aligned}
A_{n}^{(m, r)} & =\int_{0}^{1} \alpha_{m, r}(\langle x\rangle) e^{-2 \pi i n x} d x \\
& =\int_{0}^{1} \alpha_{m, r}(\langle x\rangle) e^{-2 \pi i n x} d x .
\end{aligned}
$$

For integers $m, r \geq 1$, we put

$$
\begin{aligned}
\Delta_{m, r} & =\alpha_{m, r}(1)-\alpha_{m, r}(0) \\
& =\sum_{i_{1}+\cdots+i_{2 r+1}=m}\left(P_{i_{1}}(1) P_{i_{2}}(1) \cdots P_{i_{2 r+1}}(1)-P_{i_{1}}(0) P_{i_{2}}(0) \cdots P_{i_{2 r+1}}(0)\right) .
\end{aligned}
$$

Now, from (2.8), we see that

$$
\Delta_{m, r}=\frac{1}{(2 r-1) ! ! 2^{m+r}} \sum_{k=0}^{\left[\frac{m-1}{2}\right]}(-1)^{k}\left(\begin{array}{c}
m+r \\
k
\end{array}\right)\left(\begin{array}{c}
2 m+2 r-2 k \\
m+r
\end{array}\right)(m+r-2 k)_{r} .
$$

Here we note that

$$
\alpha_{m, r}(0)= \begin{cases}\frac{(-1)^{\frac{m}{2}}}{(2 r-1) ! 2^{m+r}}\left(\begin{array}{c}
m+r \\
\frac{m}{2}
\end{array}\right)\left(\begin{array}{c}
m+2 r \\
m+r
\end{array}\right) r !, & \text { if } m \text { even } \\
0, & \text { if } m \text { odd }\end{cases}
$$

From (2.1), we observe that

$$
\begin{aligned}
\frac{d}{d x} \alpha_{m, r} & =\frac{d}{d x}\left(\frac{1}{(2 r-1) ! !} P_{m+r}^{(r)}(x)\right) \\
& =\frac{1}{(2 r-1) ! !} P_{m+r}^{(r+1)}(x) \\
& =(2 r+1) \alpha_{m-1, r+1}(x) .
\end{aligned}
$$

Thus we have shown that

$$
\frac{d}{d x} \alpha_{m, r}(x)=(2 r+1) \alpha_{m-1, r+1}(x) .
$$

Replacing $m$ by $m+1, r$ by $r-1$, from (2.15) we get

$$
\begin{aligned}
& \frac{d}{d x}\left(\frac{1}{2 r-1} \alpha_{m+1, r-1}(x)\right)=\alpha_{m, r}(x), \\
& \int_{0}^{1} \alpha_{m, r}(x) d x=\frac{1}{2 r-1} \Delta_{m+1, r-1},
\end{aligned}
$$




$$
\alpha_{m, r}(0)=\alpha_{m, r}(1) \Longleftrightarrow \Delta_{m, r}=0
$$

We are now ready to determine the Fourier coefficients $A_{n}^{(m, r)}$.

Case $1: n \neq 0$.

$$
\begin{aligned}
A_{n}^{(m, r)} & =\int_{0}^{1} \alpha_{m, r}(x) e^{-2 \pi i n x} d x \\
& =-\frac{1}{2 \pi i n}\left[\alpha_{m, r}(x) e^{-2 \pi i n x}\right]_{0}^{1}+\frac{1}{2 \pi i n} \int_{0}^{1}\left(\frac{d}{d x} \alpha_{m, r}(x)\right) e^{-2 \pi i n x} d x \\
& =-\frac{1}{2 \pi i n}\left(\alpha_{m, r}(1)-\alpha_{m, r}(0)\right)+\frac{2 r+1}{2 \pi i n} \int_{0}^{1} \alpha_{m-1, r+1}(x) e^{-2 \pi i n x} d x \\
& =\frac{2 r+1}{2 \pi i n} A_{n}^{(m-1, r+1)}-\frac{1}{2 \pi i n} \Delta_{m, r} \\
& =\frac{2 r+1}{2 \pi i n}\left(\frac{2 r+3}{2 \pi i n} A_{n}^{(m-2, r+2)}-\frac{1}{2 \pi i n} \Delta_{m-1, r+1}\right)-\frac{1}{2 \pi i n} \Delta_{m, r} \\
& =\frac{(2 r+3) ! !}{(2 \pi i n)^{2}(2 r-1) ! !} A_{n}^{(m-2, r+2)}-\sum_{j=1}^{2} \frac{(2 r+2 j-3) ! !}{(2 \pi i n)^{j}(2 r-1) ! !} \Delta_{m-j+1, r+j-1} \\
& =\cdots \\
& =\frac{(2 r+2 m-1) ! !}{(2 \pi i n)^{m}(2 r-1) ! !} A_{n}^{(0, r+m)}-\sum_{j=1}^{m} \frac{(2 r+2 j-3) ! !}{(2 \pi i n) j(2 r-1) ! !} \Delta_{m-j+1, r+j-1} \\
= & -\frac{1}{2 r-1} \sum_{j=1}^{m} \frac{(2 r+2 j-3) ! !}{(2 \pi i n)^{j}(2 r-3) ! !} \Delta_{m-j+1, r+j-1} \cdot
\end{aligned}
$$

Case 2: $n=0$.

$$
A_{0}^{(m, r)}=\int_{0}^{1} \alpha_{m, r}(x) d x=\frac{1}{2 r-1} \Delta_{m+1, r-1} .
$$

Now, from (1.7), (1.8), (2.10), (2.11), (2.19), and (2.20), we have the following Fourier series expansion of $\alpha_{m, r}(\langle x\rangle)$ :

$$
\begin{aligned}
& \frac{1}{2 r-1} \Delta_{m+1, r-1} \\
& \quad-\sum_{\substack{n=-\infty \\
n \neq 0}}^{\infty}\left(\frac{1}{2 r-1} \sum_{j=1}^{m} \frac{(2 r+2 j-3) ! !}{(2 \pi i n) j(2 r-3) ! !} \Delta_{m-j+1, r+j-1}\right) e^{2 \pi i n x} \\
& =\frac{1}{2 r-1} \Delta_{m+1, r-1} \\
& \quad+\frac{1}{2 r-1} \sum_{j=1}^{m} \frac{(2 r+2 j-3) ! !}{(2 r-3) ! ! j !} \Delta_{m-j+1, r+j-1}\left(-j ! \sum_{n=-\infty}^{\infty} \frac{e^{2 \pi i n x}}{(2 \pi i n)^{j}}\right) \\
& =\frac{1}{2 r-1} \Delta_{m+1, r-1}+\frac{1}{2 r-1} \sum_{j=2}^{m} \frac{(2 r+2 j-3) ! !}{(2 r-3) ! ! j !} \Delta_{m-j+1, r+j-1} B_{j}(\langle x\rangle) \\
& \quad+\Delta_{m, r} \times\left\{\begin{array}{l}
B_{1}(\langle x\rangle), \quad \text { for } x \notin \mathbb{Z}, \\
0, \quad \text { for } x \in \mathbb{Z}
\end{array}\right.
\end{aligned}
$$




$$
\begin{gathered}
=\frac{1}{2 r-1} \sum_{\substack{j=0 \\
j \neq 1}}^{m} \frac{(2 r+2 j-3) ! !}{(2 r-3) ! ! j !} \Delta_{m-j+1, r+j-1} B_{j}(\langle x\rangle) \\
+\Delta_{m, r} \times \begin{cases}B_{1}(\langle x\rangle), & \text { for } x \notin \mathbb{Z}, \\
0, & \text { for } x \in \mathbb{Z} .\end{cases}
\end{gathered}
$$

$\alpha_{m, r}(\langle x\rangle)(m, r \geq 1)$ is piecewise $C^{\infty}$. Moreover, $\alpha_{m, r}(\langle x\rangle)$ is continuous for those positive integers $m, r$ with $\Delta_{m, r}=0$, and discontinuous with jump discontinuities at integers for the positive integers $m, r$ with $\Delta_{m, r} \neq 0$. Thus, for $\Delta_{m, r}=0$, the Fourier series of $\alpha_{m, r}(\langle x\rangle)$ converges uniformly to $\alpha_{m, r}(\langle x\rangle)$, whereas, for $\Delta_{m, r} \neq 0$, the Fourier series of $\alpha_{m, r}(\langle x\rangle)$ converges pointwise to $\alpha_{m, r}(\langle x\rangle)$ for $x \notin \mathbb{Z}$ and converges to

$$
\begin{aligned}
\frac{1}{2}\left(\alpha_{m, r}(0)+\alpha_{m, r}(1)\right) & =\alpha_{m, r}(0)+\frac{1}{2} \Delta_{m, r} \\
& = \begin{cases}\frac{(-1)^{\frac{m}{2}}}{(2 r-1) ! 2^{m+r}}\left(\begin{array}{c}
m+r \\
\frac{m}{2}
\end{array}\right)\left(\begin{array}{c}
m+2 r \\
m+r
\end{array}\right) r !+\frac{1}{2} \Delta_{m, r}, & \text { if } m \text { even } \\
\frac{1}{2} \Delta_{m, r}, & \text { if } m \text { odd }\end{cases}
\end{aligned}
$$

for $x \in \mathbb{Z}($ see $(2.14))$.

From these observations together with (2.21) and (2.22), we obtain the next two theorems.

Theorem 2.3 For any integers $m, r$ with $m, r \geq 1$, we let

$$
\Delta_{m, r}=\frac{1}{(2 r-1) ! ! 2^{m+r}} \sum_{k=0}^{\left[\frac{m-1}{2}\right]}(-1)^{k}\left(\begin{array}{c}
m+r \\
k
\end{array}\right)\left(\begin{array}{c}
2 m+2 r-2 k \\
m+r
\end{array}\right)(m+r-2 k)_{r}
$$

Assume that $\Delta_{m, r}=0$ for some positive integers $m, r$. Then we have the following:

(a)

$$
\sum_{i_{1}+i_{2}+\cdots+i_{2 r+1}=m} P_{i_{1}}(\langle x\rangle) P_{i_{2}}(\langle x\rangle) \cdots P_{i_{2 r+1}}(\langle x\rangle)
$$

has the Fourier series expansion

$$
\begin{aligned}
& \sum_{i_{1}+i_{2}+\cdots+i_{2 r+1}=m} P_{i_{1}}(\langle x\rangle) P_{i_{2}}(\langle x\rangle) \cdots P_{i_{2 r+1}}(\langle x\rangle) \\
= & \frac{1}{2 r-1} \Delta_{m+1, r-1}-\frac{1}{2 r-1} \sum_{\substack{n=-\infty \\
n \neq 0}}^{\infty}\left(\sum_{j=1}^{m} \frac{(2 r+2 j-3) ! !}{(2 \pi i n)^{j}(2 r-3) ! !} \Delta_{m-j+1, r+j-1}\right) e^{2 \pi i n x}
\end{aligned}
$$

for all $x \in \mathbb{R}$, where the convergence is uniform.

(b)

$$
\begin{gathered}
\sum_{i_{1}+i_{2}+\cdots+i_{2 r+1}=m} P_{i_{1}}(\langle x\rangle) P_{i_{2}}(\langle x\rangle) \cdots P_{i_{2 r+1}}(\langle x\rangle) \\
=\frac{1}{2 r-1} \sum_{\substack{j=0 \\
j \neq 1}}^{m} \frac{(2 r+2 j-3) ! !}{(2 r-3) ! ! j !} \Delta_{m-j+1, r+j-1} B_{j}(\langle x\rangle)
\end{gathered}
$$

for all $x \in \mathbb{R}$. 
Theorem 2.4 For any integers $m, r$ with $m, r \geq 1$, we let

$$
\Delta_{m, r}=\frac{1}{(2 r-1) ! ! 2^{m+r}} \sum_{k=0}^{\left[\frac{m-1}{2}\right]}(-1)^{k}\left(\begin{array}{c}
m+r \\
k
\end{array}\right)\left(\begin{array}{c}
2 m+2 r-2 k \\
m+r
\end{array}\right)(m+r-2 k)_{r}
$$

Assume that $\Delta_{m, r} \neq 0$ for some positive integers $m, r$. Then we have the following:

(a)

$$
\begin{aligned}
& \frac{1}{2 r-1} \Delta_{m+1, r-1}-\frac{1}{2 r-1} \sum_{\substack{n=-\infty \\
n \neq 0}}^{\infty}\left(\sum_{j=1}^{m} \frac{(2 r+2 j-3) ! !}{(2 \pi i n)^{j}(2 r-3) ! !} \Delta_{m-j+1, r+j-1}\right) e^{2 \pi i n x} \\
& \quad= \begin{cases}\sum_{i_{1}+\cdots+i_{2 r+1}=m} P_{i_{1}}(\langle x\rangle) P_{i_{2}}(\langle x\rangle) \cdots P_{i_{2 r+1}}(\langle x\rangle), & \text { for } x \notin \mathbb{Z}, \\
\frac{(-1)^{\frac{m}{2}}}{(2 r-1) ! 2^{m+r}}\left(\begin{array}{c}
m+r \\
\frac{m}{2}
\end{array}\right)\left(\begin{array}{c}
m+2 r \\
m+r
\end{array}\right) r !+\frac{1}{2} \Delta_{m, r}, & \text { for } x \in \mathbb{Z} \text { and } m \text { even }, \\
\frac{1}{2} \Delta_{m, r}, & \text { for } x \in \mathbb{Z} \text { and } m \text { odd. } .\end{cases}
\end{aligned}
$$

(b)

$$
\begin{aligned}
& \frac{1}{2 r-1} \sum_{j=0}^{m} \frac{(2 r+2 j-3) ! !}{(2 r-3) ! ! j !} \Delta_{m-j+1, r+j-1} B_{j}(\langle x\rangle) \\
& =\sum_{i_{1}+i_{2}+\cdots+i_{2 r+1}=m} P_{i_{1}}(\langle x\rangle) P_{i_{2}}(\langle x\rangle) \cdots P_{i_{2 r+1}}(\langle x\rangle), \quad \text { for } x \notin \mathbb{Z} ; \\
& \frac{1}{2 r-1} \sum_{\substack{j=0 \\
j \neq 1}}^{m} \frac{(2 r+2 j-3) ! !}{(2 r-3) ! ! j !} \Delta_{m-j+1, r+j-1} B_{j}(\langle x\rangle) \\
& \quad= \begin{cases}\frac{(-1)^{\frac{m}{2}}}{(2 r-1) ! 2^{m+r}}\left(\begin{array}{c}
m+r \\
\frac{m}{2}
\end{array}\right)\left(\begin{array}{c}
m+2 r \\
m+r
\end{array}\right) r !+\frac{1}{2} \Delta_{m, r}, & \text { if m even, } \\
\frac{1}{2} \Delta_{m, r}, & \text { if m odd },\end{cases}
\end{aligned}
$$

for $x \in \mathbb{Z}$.

Finally, we observe that the statement in Theorem A follows immediately from Theorems 2.3 and 2.4 .

\section{Fourier series expansions for functions associated with Laguerre polynomials}

The following lemma is needed for our discussion in this section.

Lemma 3.1 Let $n, r$ be integers with $n, r \geq 0$. Then we have the identity

$$
\sum_{i_{1}+i_{2}+\cdots+i_{r+1}=n} L_{i_{1}}\left(\frac{x}{r+1}\right) L_{i_{2}}\left(\frac{x}{r+1}\right) \cdots L_{i_{r+1}}\left(\frac{x}{r+1}\right)=(-1)^{r} L_{n+r}^{(r)}(x),
$$

where the sum runs over all nonnegative integers $i_{1}, i_{2}, \ldots, i_{r+1}$ with $i_{1}+i_{2}+\cdots+i_{r+1}=n$.

Proof Differentiating (1.4) $r$ times, we get

$$
\frac{\partial^{r} G(t, x)}{\partial x^{r}}=(-1)^{r} t^{r}(1-t)^{-r-1} \exp \left(-\frac{x t}{1-t}\right)
$$




$$
\frac{\partial^{r} G(t, x)}{\partial x^{r}}=\sum_{n=r}^{\infty} L_{n}^{(r)}(x) t^{n}=\sum_{n=0}^{\infty} L_{n+r}^{(r)}(x) t^{n+r}
$$

Equating (3.2) and (3.3) gives us

$$
(-1)^{r}(1-t)^{-r-1} \exp \left(-\frac{x t}{1-t}\right)=\sum_{n=0}^{\infty} L_{n+r}^{(r)} t^{n}
$$

On the other hand, from (1.4) and (3.4), we note that

$$
\begin{aligned}
& \sum_{n=0}^{\infty}\left(\sum_{i_{1}+i_{2}+\cdots+i_{r+1}=n} L_{i_{1}}\left(\frac{x}{r+1}\right) L_{i_{2}}\left(\frac{x}{r+1}\right) \cdots L_{i_{r+1}}\left(\frac{x}{r+1}\right)\right) t^{n} \\
& \quad=\left(\sum_{n=0}^{\infty} L_{n}\left(\frac{x}{r+1}\right) t^{n}\right)^{r+1} \\
& =\left((1-t)^{-1} \exp \left(-\frac{x t}{(r+1)(1-t)}\right)\right)^{r+1} \\
& =(-1)^{r} \sum_{n=0}^{\infty} L_{n+r}^{(r)} t^{n} .
\end{aligned}
$$

Comparing both sides of (3.5) yields the desired result.

It is known that the Laguerre polynomials $L_{n}(x)$ are given by (see $\left.[2,3]\right)$

$$
L_{n}(x)={ }_{1} F_{1}\left(\begin{array}{c}
-n \\
1
\end{array} \mid x\right)=\sum_{k=0}^{n}(-1)^{k}\left(\begin{array}{l}
n \\
k
\end{array}\right) \frac{1}{k !} x^{k}
$$

where ${ }_{1} F_{1}\left({ }_{b}^{a} \mid z\right)=\sum_{n=0}^{\infty} \frac{\langle a\rangle_{n}}{\langle b\rangle_{n}} \frac{z^{n}}{n !}$ is the hypergeometric function.

The $r$ th derivative of (3.6) is given by

$$
\begin{aligned}
L_{n}^{(r)}(x) & =\sum_{k=r}^{n}(-1)^{k} \frac{1}{k !}\left(\begin{array}{l}
n \\
k
\end{array}\right)(k)_{r} x^{k-r} \\
& =\sum_{k=r}^{n}(-1)^{k} \frac{1}{(k-r) !}\left(\begin{array}{l}
n \\
k
\end{array}\right) x^{k-r} \\
& =\sum_{k=0}^{n-r}(-1)^{k+r} \frac{1}{k !}\left(\begin{array}{c}
n \\
k+r
\end{array}\right) x^{k} \quad(0 \leq r \leq n) .
\end{aligned}
$$

From (3.1) and (3.7), we obtain the following result.

Lemma 3.2 For integers $n, r$ with $n, r \geq 0$, we have the following identity:

$$
\begin{aligned}
& \quad \sum_{i_{1}+i_{2}+\cdots+i_{r+1}=n} L_{i_{1}}\left(\frac{x}{r+1}\right) L_{i_{2}}\left(\frac{x}{r+1}\right) \cdots L_{i_{r+1}}\left(\frac{x}{r+1}\right) \\
& =\sum_{k=0}^{n} \frac{(-1)^{k}}{k !}\left(\begin{array}{l}
n+r \\
k+r
\end{array}\right) x^{k} .
\end{aligned}
$$


For integers $m, r$ with $m \geq 1, r \geq 0$ as in (1.11), we put

$$
\beta_{m, r}(x)=\sum_{i_{1}+i_{2}+\cdots+i_{r+1}=m} L_{i_{1}}\left(\frac{x}{r+1}\right) L_{i_{2}}\left(\frac{x}{r+1}\right) \cdots L_{i_{r+1}}\left(\frac{x}{r+1}\right) .
$$

Then we will consider the function

$$
\beta_{m, r}(\langle x\rangle)=\sum_{i_{1}+i_{2}+\cdots+i_{r+1}=m} L_{i_{1}}\left(\frac{\langle x\rangle}{r+1}\right) L_{i_{2}}\left(\frac{\langle x\rangle}{r+1}\right) \cdots L_{i_{r+1}}\left(\frac{\langle x\rangle}{r+1}\right)
$$

defined on $\mathbb{R}$, which is periodic with period 1 .

The Fourier series of $\beta_{m, r}(\langle x\rangle)$ is

$$
\sum_{n=-\infty}^{\infty} B_{n}^{(m, r)} e^{2 \pi i n x}
$$

where

$$
\begin{aligned}
B_{n}^{(m, r)} & =\int_{0}^{1} \beta_{m, r}(\langle x\rangle) e^{-2 \pi i n x} d x \\
& =\int_{0}^{1} \beta_{m, r}(x) e^{-2 \pi i n x} d x
\end{aligned}
$$

For integers $m, r$ with $m \geq 1, r \geq 0$, we set

$$
\begin{aligned}
\Omega_{m, r}= & \beta_{m, r}(1)-\beta_{m, r}(0) \\
= & \sum_{i_{1}+i_{2}+\cdots+i_{r+1}=m}\left(L_{i_{1}}\left(\frac{1}{r+1}\right) L_{i_{2}}\left(\frac{1}{r+1}\right) \cdots L_{i_{r+1}}\left(\frac{1}{r+1}\right)\right. \\
& \left.-L_{i_{1}}(0) L_{i_{2}}(0) \cdots L_{i_{r+1}}(0)\right) .
\end{aligned}
$$

Now, from (3.8), we see that

$$
\Omega_{m, r}=(-1)^{m} \sum_{k=0}^{m-1} \frac{(-1)^{k}}{(m-k) !}\left(\begin{array}{c}
m+r \\
k
\end{array}\right)
$$

From (3.1), we note that

$$
\begin{aligned}
\frac{d}{d x} \beta_{m, r}(x) & =\frac{d}{d x}\left((-1)^{r} L_{m+r}^{(r)}(x)\right) \\
& =(-1)^{r} L_{m+r}^{(r+1)}(x) \\
& =-\beta_{m-1, r+1}(x) .
\end{aligned}
$$

Thus we have shown that

$$
\frac{d}{d x} \beta_{m, r}(x)=-\beta_{m-1, r+1}(x)
$$


Replacing $m$ by $m+1, r$ by $r-1$, from (3.14), we get

$$
\begin{aligned}
& \frac{d}{d x}\left(-\beta_{m+1, r-1}(x)\right)=\beta_{m, r}(x), \\
& \int_{0}^{1} \beta_{m, r}(x) d x=-\Omega_{m+1, r-1}, \\
& \beta_{m, r}(0)=\beta_{m, r}(1) \quad \Longleftrightarrow \quad \Omega_{m, r}=0 .
\end{aligned}
$$

We are now going to determine the Fourier coefficients.

Case $1: n \neq 0$.

$$
\begin{aligned}
B_{n}^{(m, r)} & =\int_{0}^{1} \beta_{m, r}(x) e^{-2 \pi i n x} d x \\
& =-\frac{1}{2 \pi i n}\left[\beta_{m, r}(x) e^{-2 \pi i n x}\right]_{0}^{1}+\frac{1}{2 \pi i n} \int_{0}^{1}\left(\frac{d}{d x} \beta_{m, r}(x)\right) e^{-2 \pi i n x} d x \\
& =-\frac{1}{2 \pi i n}\left(\beta_{m, r}(1)-\beta_{m, r}(0)\right)-\frac{1}{2 \pi i n} \int_{0}^{1} \beta_{m-1, r+1}(x) e^{-2 \pi i n x} d x \\
& =-\frac{1}{2 \pi i n} B_{n}^{(m-1, r+1)}-\frac{1}{2 \pi i n} \Omega_{m, r} \\
& =-\frac{1}{2 \pi i n}\left(-\frac{1}{2 \pi i n} B_{n}^{(m-2, r+2)}-\frac{1}{2 \pi i n} \Omega_{m-1, r+1}\right)-\frac{1}{2 \pi i n} \Omega_{m, r} \\
& =\left(-\frac{1}{2 \pi i n}\right)^{2} B_{n}^{(m-2, r+2)}+\sum_{j=1}^{2}\left(-\frac{1}{2 \pi i n}\right)^{j} \Omega_{m-j+1, r+j-1} \\
& =\cdots \\
& =\left(-\frac{1}{2 \pi i n}\right)^{m} B_{n}^{(0, r+m)}+\sum_{j=1}^{m}\left(-\frac{1}{2 \pi i n}\right)^{j} \Omega_{m-j+1, r+j-1} \\
& =\sum_{j=1}^{m}\left(-\frac{1}{2 \pi i n}\right)^{j} \Omega_{m-j+1, r+j-1} .
\end{aligned}
$$

Case 2: $n=0$.

$$
B_{0}^{(m, r)}=\int_{0}^{1} \beta_{m, r}(x) d x=-\Omega_{m+1, r-1} .
$$

Now, from (1.7), (1.8), (3.10), (3.11), (3.18), and (3.19), the Fourier series expansion of $\beta_{m, r}(\langle x\rangle)$ is given by

$$
\begin{aligned}
& -\Omega_{m+1, r-1}+\sum_{\substack{n=-\infty \\
n \neq 0}}^{\infty}\left(\sum_{j=1}^{m}\left(-\frac{1}{2 \pi i n}\right)^{j} \Omega_{m-j+1, r+j-1}\right) e^{2 \pi i n x} \\
& =-\Omega_{m+1, r-1}-\sum_{j=1}^{m} \frac{(-1)^{j}}{j !} \Omega_{m-j+1, r+j-1}\left(-j ! \sum_{\substack{n=-\infty \\
n \neq 0}}^{\infty} \frac{e^{2 \pi i n x}}{(2 \pi i n)^{j}}\right) \\
& =-\Omega_{m+1, r-1}-\sum_{j=2}^{m} \frac{(-1)^{j}}{j !} \Omega_{m-j+1, r+j-1} B_{j}(\langle x\rangle)
\end{aligned}
$$




$$
\begin{gathered}
+\Omega_{m, r} \times \begin{cases}B_{1}(\langle x\rangle), & \text { if } x \notin \mathbb{Z}, \\
0, & \text { if } x \in \mathbb{Z}\end{cases} \\
=-\sum_{\substack{j=0 \\
j \neq 1}}^{m} \frac{(-1)^{j}}{j !} \Omega_{m-j+1, r+j-1} B_{j}(\langle x\rangle)+\Omega_{m, r} \times \begin{cases}B_{1}(\langle x\rangle), & \text { if } x \notin \mathbb{Z}, \\
0, & \text { if } x \in \mathbb{Z} .\end{cases}
\end{gathered}
$$

$\beta_{m, r}(\langle x\rangle)(m \geq 1, r \geq 0)$ is piecewise $C^{\infty}$. In addition, $\beta_{m, r}(\langle x\rangle)$ is continuous for those integers $m, r(m \geq 1, r \geq 0)$ with $\Omega_{m, r}=0$, and discontinuous with jump discontinuities at integers for those integers $m, r(m \geq 1, r \geq 0)$ with $\Omega_{m, r} \neq 0$. Hence, for $\Omega_{m, r}=0$, the Fourier series of $\beta_{m, r}(\langle x\rangle)$ converges uniformly to $\beta_{m, r}(\langle x\rangle)$. Whereas, for $\Omega_{m, r} \neq 0$, the Fourier series of $\beta_{m, r}(\langle x\rangle)$ converges pointwise to $\beta_{m, r}(\langle x\rangle)$ for $x \notin \mathbb{Z}$ and converges to

$$
\frac{1}{2}\left(\beta_{m, r}(0)+\beta_{m, r}(1)\right)=\beta_{m, r}(0)+\frac{1}{2} \Omega_{m, r}=\left(\begin{array}{c}
m+r \\
r
\end{array}\right)+\frac{1}{2} \Omega_{m, r}
$$

for $x \in \mathbb{Z}$ (see (3.8)).

From these observations together with (3.20) and (3.21), we have the next two theorems.

Theorem 3.3 For any integers $m, r$ with $m \geq 1, r \geq 0$, we let

$$
\Omega_{m, r}=(-1)^{m} \sum_{k=0}^{m-1} \frac{(-1)^{k}}{(m-k) !}\left(\begin{array}{c}
m+r \\
k
\end{array}\right)
$$

Assume that $\Omega_{m, r}=0$ for some integers $m, r$ with $m \geq 1, r \geq 0$. Then we have the following:

(a)

$$
\sum_{i_{1}+i_{2}+\cdots+i_{r+1}=m} L_{i_{1}}\left(\frac{\langle x\rangle}{r+1}\right) L_{i_{2}}\left(\frac{\langle x\rangle}{r+1}\right) \cdots L_{i_{r+1}}\left(\frac{\langle x\rangle}{r+1}\right)
$$

has the Fourier series expansion

$$
\begin{gathered}
\sum_{i_{1}+i_{2}+\cdots+i_{r+1}=m} L_{i_{1}}\left(\frac{\langle x\rangle}{r+1}\right) L_{i_{2}}\left(\frac{\langle x\rangle}{r+1}\right) \cdots L_{i_{r+1}}\left(\frac{\langle x\rangle}{r+1}\right) \\
=-\Omega_{m+1, r-1}+\sum_{\substack{n=-\infty \\
n \neq 0}}^{\infty}\left(\sum_{j=1}^{m}\left(-\frac{1}{2 \pi i n}\right)^{j} \Omega_{m-j+1, r+j-1}\right) e^{2 \pi i n x}
\end{gathered}
$$

for all $x \in \mathbb{R}$, where the convergence is uniform.

(b)

$$
\begin{aligned}
& \sum_{i_{1}+i_{2}+\cdots+i_{r+1}=m} L_{i_{1}}\left(\frac{\langle x\rangle}{r+1}\right) L_{i_{2}}\left(\frac{\langle x\rangle}{r+1}\right) \cdots L_{i_{r+1}}\left(\frac{\langle x\rangle}{r+1}\right) \\
& =-\sum_{\substack{j=0 \\
j \neq 1}}^{m} \frac{(-1)^{j}}{j !} \Omega_{m-j+1, r+j-1} B_{j}(\langle x\rangle)
\end{aligned}
$$

for all $x \in \mathbb{R}$. 
Theorem 3.4 For any integers $m, r$ with $m \geq 1, r \geq 0$, we let

$$
\Omega_{m, r}=(-1)^{m} \sum_{k=0}^{m-1} \frac{(-1)^{k}}{(m-k) !}\left(\begin{array}{c}
m+r \\
k
\end{array}\right) .
$$

Assume that $\Omega_{m, r} \neq 0$ for some integers $m, r$ with $m \geq 1, r \geq 0$. Then we have the following:

(a)

$$
\begin{array}{r}
-\Omega_{m+1, r-1}+\sum_{\substack{n=-\infty \\
n \neq 0}}^{\infty}\left(\sum_{j=1}^{m}\left(-\frac{1}{2 \pi i n}\right)^{j} \Omega_{m-j+1, r+j-1}\right) e^{2 \pi i n x} \\
= \begin{cases}\sum_{i_{1}+i_{2}+\cdots+i_{r+1}=m} L_{i_{1}}\left(\frac{\langle x\rangle}{r+1}\right) L_{i_{2}}\left(\frac{\langle x\rangle}{r+1}\right) \cdots L_{i_{r+1}}\left(\frac{\langle x\rangle}{r+1}\right), & \text { for } x \notin \mathbb{Z}, \\
\left(\begin{array}{c}
m+r \\
r
\end{array}\right)+\frac{1}{2} \Omega_{m, r}, & \text { for } x \in \mathbb{Z} .\end{cases}
\end{array}
$$

(b)

$$
\begin{aligned}
& -\sum_{j=0}^{m} \frac{(-1)^{j}}{j !} \Omega_{m-j+1, r+j-1} B_{j}(\langle x\rangle) \\
& =\sum_{i_{1}+i_{2}+\cdots+i_{r+1}=m} L_{i_{1}}\left(\frac{\langle x\rangle}{r+1}\right) L_{i_{2}}\left(\frac{\langle x\rangle}{r+1}\right) \cdots L_{i_{r+1}}\left(\frac{\langle x\rangle}{r+1}\right), \quad \text { for } x \notin \mathbb{Z} \\
& -\sum_{\substack{j=0 \\
j \neq 1}}^{m} L_{i_{1}}\left(\frac{\langle x\rangle}{r+1}\right) L_{i_{2}}\left(\frac{\langle x\rangle}{r+1}\right) \cdots L_{i_{r+1}}\left(\frac{\langle x\rangle}{r+1}\right) \\
& =\left(\begin{array}{c}
m+r \\
r
\end{array}\right)+\frac{1}{2} \Omega_{m, r}, \quad \text { for } x \in \mathbb{Z} .
\end{aligned}
$$

Finally, we see that the statement in Theorem B follows from Theorems 3.3 and 3.4.

\section{Expressions in terms of Euler polynomials}

For any polynomial $p(x) \in \mathbb{C}[x]$ with degree $m$, we know that

$$
p(x)=\sum_{k=0}^{m} b_{k} E_{k}(x)
$$

where $E_{k}(x)$ are the Euler polynomials given by $\frac{2}{e^{t}+1} e^{x t}=\sum_{k=0}^{\infty} E_{k}(x) \frac{t^{k}}{k !}$, and

$$
b_{k}=\frac{1}{2 k !}\left(p^{(k)}(1)+p^{(k)}(0)\right), \quad k=0,1, \ldots, m
$$

Applying (4.1) and (4.2) to $p(x)=\alpha_{m, r}(x)$ and from (2.15), we see that

$$
\alpha_{m, r}^{(k)}(x)=\frac{(2 r+2 k-1) ! !}{(2 r-1) ! !} \alpha_{m-k, r+k}(x)
$$


Hence, from (4.3), we have

$$
\begin{aligned}
b_{k} & =\frac{1}{2 k !}\left(\alpha_{m, r}^{(k)}(1)+\alpha_{m, r}^{(k)}(0)\right) \\
& =\frac{(2 r+2 k-1) ! !}{2 k !(2 r-1) ! !}\left(\alpha_{m-k, r+k}(1)+\alpha_{m-k, r+k}(0)\right) \\
& =\frac{(2 r+2 k-1) ! !}{k !(2 r-1) ! !}\left(\alpha_{m-k, r+k}(0)+\frac{1}{2} \Delta_{m-k, r+k}\right) .
\end{aligned}
$$

Now, from (2.14), (4.1), and (4.4), we get the following theorem.

Theorem 4.1 For any integers $m, r$ with $m, r \geq 1$, we have the following:

$$
\sum_{i_{1}+i_{2}+\cdots+i_{2 r+1}=m} P_{i_{1}}(x) P_{i_{2}}(x) \cdots P_{i_{2 r+1}}(x)=\sum_{k=0}^{m} b_{k} E_{k}(x)
$$

where

$$
b_{k}= \begin{cases}\frac{(-1)^{\frac{m-k}{2}}(r+k) !}{k !(2 r-1) ! ! 2^{m+r}}\left(\begin{array}{c}
m+r \\
\frac{m-k}{2}
\end{array}\right)\left(\begin{array}{c}
m+2 r+k \\
m+r
\end{array}\right)+\frac{(2 r+2 k-1) ! !}{2 k !(2 r-1) ! !} \Delta_{m-k, r+k}, & \text { for } m-k \text { even }, \\
\frac{(2 r+2 k-1) ! !}{2 k !(2 r-1) ! !} \Delta_{m-k, r+k}, & \text { for } m-k \text { odd },\end{cases}
$$

and $\Delta_{m, r}$ is given by (2.13).

Proceeding analogously to the above discussion, we get the next result, the details of which are left to the reader.

Theorem 4.2 For any integers $m, r$ with $m \geq 1, r \geq 0$, we have the following identity:

$$
\begin{aligned}
& \sum_{i_{1}+i_{2}+\cdots+i_{r+1}=m} L_{i_{1}}\left(\frac{x}{r+1}\right) L_{i_{2}}\left(\frac{x}{r+1}\right) \cdots L_{i_{r+1}}\left(\frac{x}{r+1}\right) \\
= & \sum_{k=0}^{m} \frac{(-1)^{k}}{k !}\left(\left(\begin{array}{c}
m+r \\
r+k
\end{array}\right)+\frac{1}{2} \Omega_{m-k, r+k}\right) E_{k}(x),
\end{aligned}
$$

where $\Omega_{m, r}$ is given by (3.13).

\section{Results and discussion}

In this paper, we investigated sums of finite products of Legendre and Laguerre polynomials and derived Fourier series expansions of functions associated with those polynomials. From these Fourier series expansions, we were able to express those sums of finite products as linear combinations of Bernoulli polynomials. Further, by using a different method, we expressed those sums of finite products as linear combinations of Euler polynomials as well. It is expected that we will be able to express those sums of finite products as linear combinations of some classical orthogonal polynomials. Here we note that the Bernoulli and Euler polynomials are not orthogonal polynomials but Appell polynomials. 


\section{Conclusion}

In this paper, we considered the Fourier series expansions for functions associated with Legendre and Laguerre polynomials. In addition, by using a method other than Fourier series expansions, we were able to express those sums in terms of Euler polynomials. It is noteworthy that all the other previous related papers, except [5, 20-22], were associated with Appell polynomials, while the present one is about classical orthogonal polynomials.

Funding

This research was supported by the Basic Science Research Program through the National Research Foundation of Korea (NRF) funded by the Ministry of Education (No. NRF-2017R1D1A1B03034892).

\section{Competing interests}

The authors declare that they have no competing interests.

\section{Authors' contributions}

All authors contributed equally to the manuscript and typed, read, and approved the final manuscript.

\section{Author details}

'Department of Mathematics, Kwangwoon University, Seoul, Republic of Korea. ${ }^{2}$ Department of Mathematics, Sogang University, Seoul, Republic of Korea. ${ }^{3}$ Hanrimwon, Kwangwoon University, Seoul, Republic of Korea. ${ }^{4}$ Department of Mathematics Education, Daegu University, Gyeongsan-si, Republic of Korea.

\section{Publisher's Note}

Springer Nature remains neutral with regard to jurisdictional claims in published maps and institutional affiliations.

Received: 4 June 2018 Accepted: 31 July 2018 Published online: 10 August 2018

\section{References}

1. Cesarano, C., Ricci, P.E.: The Legendre polynomials as a basis for Bessel functions. Int. J. Pure Appl. Math. 111, 129-140 (2016)

2. Andrews, G.E., Askey, R., Roy, R.: Special Functions. Encyclopedia of Mathematics and Its Applications, vol. 71. Cambridge University Press, Cambridge (1999)

3. Beals, R., Wang, R.: Special Functions: A Graduate Text. Cambridge Studies in Advanced Mathematics, vol. 126. Cambridge University Press, Cambridge (2010)

4. Dattoli, G., Srivastava, H.M., Cesarano, C.: The Laguerre and Legendre polynomials from an operational point of view. Appl. Math. Comput. 124(1), 117-127 (2001)

5. Kim, T., Kim, D.S., Dolgy, D.V., Park, J.W.: Sums of finite products of Chebyshev polynomials of the second kind and of Fibonacci polynomials. J. Inequal. Appl. 2018, 148 (2018)

6. Agarwal, R.P., Kim, D.S., Kim, T., Kwon, J.: Sums of finite products of Bernoulli functions. Adv. Differ. Equ. 2017, 237 (2017)

7. Kim, T., Kim, D.S., Jang, G.-W., Kwon, J.: Sums of finite products of Euler functions. In: Advances in Real and Complex Analysis with Applications. Trends in Math., pp. 243-260. Springer, Berlin (2017)

8. Kim, T., Kim, D.S., Jang, G.-W., Kwon, J.: Fourier series of finite products of Bernoulli and Genocchi functions. J. Inequal. Appl. 2017, 157 (2017)

9. Kim, T., Kim, D.S., Jang, L.C., Jang, G.-W.: Sums of finite products of Genocchi functions. Adv. Differ. Equ. 2017, 268 (2017)

10. Faber, C., Pandharipande, R.: Hodge integrals and Gromov-Witten theory. Invent. Math. 139, 173-199 (2000)

11. Dunne, G.V., Schubert, C.: Bernoulli number identities from quantum field theory and topological string theory. Commun. Number Theory Phys. 7, 225-249 (2013)

12. Gessel, I.M.: On Miki's identity for Bernoulli numbers. J. Number Theory 110, 75-82 (2005)

13. Miki, H.: A relation between Bernoulli numbers. J. Number Theory 10, 297-302 (1978)

14. Shiratani, K., Yokoyama, S.: An application of p-adic convolutions. Mem. Fac. Sci., Kyushu Univ., Ser. A, Math. 36, 73-83 (1982)

15. Dattoli, G., Lorenzutta, S., Cesarano, C.: Bernstein polynomials and operational methods. J. Comput. Anal. Appl. 8, 369-377 (2006)

16. Dattoli, G., Lorenzutta, S., Ricci, P.E., Cesarano, C.: On a family of hybrid polynomials. Integral Transforms Spec. Funct. $15,485-490(2004)$

17. Bayad, A., Navas, L.: Algebraic properties and Fourier expansions of two-dimensional Apostol-Bernoulli and Apostol-Euler polynomials. Appl. Math. Comput. 265, 883-892 (2015)

18. Simsek, Y.: Combinatorial identities associated with Bernstein type basis functions. Filomat 30(7), 1683-1689 (2016)

19. Simsek, Y.: Formulas for Poisson-Charlier, Hermite, Milne-Thomson and other type polynomials by their generating functions and $p$-adic integral approach. Rev. R. Acad. Cienc. Exactas Fís. Nat., Ser. A Mat., 1-18 (to appear). https://doi.org/10.1007/s13398-018-0528-6

20. Kim, T., Kim, D.S., Dolgy, D.V., Ryoo, C.S.: Representing sums of finite products of Chebyshev polynomials of third and fourth kinds by Chebyshev polynomials. Symmetry 10(7), 258 (2018) 
21. Kim, D.S., Kim, T., Kwon, H.I., Kwon, J.: Fourier series of sums of products of higher-order Genocchi functions. Adv. Stud. Contemp. Math. (Kyungshang) 28(2), 215-230 (2018)

22. Kim, D.S., Kim, T., Kwon, H.-I.: Fourier series of $r$-derangement and higher-order derangement functions. Adv. Stud. Contemp. Math. (Kyungshang) 28(1), 1-11 (2018)

Submit your manuscript to a SpringerOpen ${ }^{\circ}$ journal and benefit from:

- Convenient online submission

$\checkmark$ Rigorous peer review

Open access: articles freely available online

- High visibility within the field

- Retaining the copyright to your article

Submit your next manuscript at $\gg$ springeropen.com 